\title{
SIX-DIMENSIONAL EXCEPTIONAL QUOTIENT SINGULARITIES
}

\author{
Ivan Cheltsov and Constantin Shramov
}

\begin{abstract}
We classify six-dimensional exceptional quotient singularities and show that seven-dimensional exceptional quotient singularities do not exist. Inter alia we prove that the irreducible six-dimensional projective representation of the sporadic simple Hall-Janko group gives rise to an exceptional quotient singularity.
\end{abstract}

We assume that all varieties are projective, normal, and defined over $\mathbb{C}$.

\section{Introduction}

Let $(V \ni O)$ be a germ of a Kawamata log terminal singularity (see [13, Definition 3.5]), and let $\xi: \bar{V} \rightarrow V$ be a resolution of singularities of the variety $V$. Then

$$
K_{\bar{V}} \sim_{\mathbb{Q}} \xi^{*}\left(K_{V}\right)+\sum_{i=1}^{r} b_{i} E_{i}
$$

where $E_{i}$ is a $\xi$-exceptional divisor, and $b_{i} \in \mathbb{Q}$. Let $B$ be an effective $\mathbb{Q}$-divisor on $V$. Put

$$
B=\sum_{i=1}^{m} a_{i} B_{i}
$$

where $B_{i}$ is a prime Weil divisor on $V$, and $a_{i} \in \mathbb{Q} \geqslant 0$. Suppose that $B$ is a $\mathbb{Q}$-Cartier divisor. Then

$$
\sum_{i=1}^{m} a_{i} \bar{B}_{i} \sim_{\mathbb{Q}} \xi^{*}\left(\sum_{i=1}^{m} a_{i} B_{i}\right)-\sum_{i=1}^{r} c_{i} E_{i}
$$

where $\bar{B}_{i}$ is the proper transform of the divisor $B_{i}$ on the variety $\bar{V}$. Suppose that

$$
\left(\bigcup_{i=1}^{m} \bar{B}_{i}\right) \bigcup\left(\bigcup_{i=1}^{r} E_{i}\right)
$$

is a divisor with simple normal crossing.

Definition 1.1 ([13, Definition 8.1]). The log canonical threshold of the divisor $B$ at $O$ is

$$
\mathrm{c}_{O}(X, B)=\min \left(\min \left\{\frac{1}{a_{i}} \mid O \in B_{i}\right\}, \min \left\{\frac{b_{i}+1}{c_{i}} \mid O \in \xi\left(E_{i}\right)\right\}\right) \in \mathbb{Q}_{>0} \cup\{+\infty\} .
$$

Received by the editors April 5, 2010. 
Definition 1.2 ([8, Definition 2.3.1]). One defines the log canonical multiplicity $\mu=\mu_{O}(X, B) \in \mathbb{Z}_{\geqslant 0}$ of the divisor $B$ at $O$ as

$$
\mu=\max \left\{\begin{array}{l|l}
\alpha+\beta & \begin{array}{l}
O \in \xi\left(\left(\bar{B}_{i_{1}} \cap \cdots \cap \bar{B}_{i_{\alpha}}\right) \cap\left(E_{k_{1}} \cap \cdots \cap E_{k_{\beta}}\right)\right) \text { and } \\
\frac{1}{a_{i_{1}}}=\cdots=\frac{1}{a_{i_{\alpha}}}=\frac{b_{k_{1}}+1}{c_{k_{1}}}=\cdots=\frac{b_{k_{\beta}}+1}{c_{k_{\beta}}}=\mathrm{c}_{O}(X, B)
\end{array}
\end{array}\right\}
$$

One can show that the numbers $\mathrm{c}_{O}(X, B)$ and $\mu_{O}(X, B)$ do not depend on the choice of the log resolution $\xi$.

Definition 1.3 ([20, Definition 2.5]). The singularity $(V \ni O)$ is exceptional if

$$
\mu_{O}(X, B) \leqslant 1
$$

for every effective $\mathbb{Q}$-Cartier $\mathbb{Q}$-divisor $B$ on the variety $V$.

Note that Definition 1.3 looks different from [20, Definition 2.5], but they are equivalent. One can show that exceptional singularities exist in any dimension greater than 1 (see [7, Example 3.13]).

Example 1.1. Suppose that $\operatorname{dim}(V)=2$ and $-K_{V}$ is Cartier. Then the singularity $(V \ni O)$ is exceptional if and only if it is a Du Val singularity of type $\mathbb{E}_{6}, \mathbb{E}_{7}$ or $\mathbb{E}_{8}$.

Let $G$ be a finite subgroup in $\mathrm{GL}_{n+1}(\mathbb{C})$, where $n \geqslant 1$. Put

$$
\bar{G}=\phi(G) \subset \operatorname{Aut}\left(\mathbb{P}^{n}\right) \cong \operatorname{PGL}_{n+1}(\mathbb{C}),
$$

where $\phi: \mathrm{GL}_{n+1}(\mathbb{C}) \rightarrow \operatorname{Aut}\left(\mathbb{P}^{n}\right)$ is the natural projection. Put

$$
\operatorname{lct}\left(\mathbb{P}^{n}, \bar{G}\right)=\sup \left\{\begin{array}{l|l}
\lambda \in \mathbb{Q} & \begin{array}{l}
\text { the log pair }\left(\mathbb{P}^{n}, \lambda D\right) \text { has log canonical } \\
\text { singularities for every } \bar{G} \text {-invariant effective } \\
\mathbb{Q} \text {-divisor } D \sim_{\mathbb{Q}}-K_{\mathbb{P}^{n}}
\end{array}
\end{array}\right\} \in \mathbb{R} \text {. }
$$

Remark 1.1 (cf. Appendix A). It follows from [6, Theorem A.3] that

$$
\operatorname{lct}\left(\mathbb{P}^{n}, \bar{G}\right)=\alpha_{\bar{G}}\left(\mathbb{P}^{n}\right),
$$

where $\alpha_{\bar{G}}\left(\mathbb{P}^{n}\right)$ is the $\bar{G}$-invariant $\alpha$-invariant introduced in [30, 31].

We are going to study the quotient singularity $\mathbb{C}^{n+1} / G$.

Remark 1.2. Let $R \subseteq G$ be a subgroup generated by all reflections in $G$ (see [28, Section 4.1]). Then the quotient $\mathbb{C}^{n+1} / R$ is isomorphic to $\mathbb{C}^{n+1}$ (see $[28$, Theorem 4.2.5]). Moreover, the subgroup $R \subseteq G$ is normal, and the singularity $\mathbb{C}^{n+1} / G$ is isomorphic to the singularity $\mathbb{C}^{n+1} /(G / R)$. Note that the subgroup $R$ is trivial if $G \subset \mathrm{SL}_{n+1}(\mathbb{C})$. If $G=R$ (in particular, if $G$ is a trivial group), then the singularity $\mathbb{C}^{n+1} / G \cong \mathbb{C}^{n+1}$ is not exceptional.

The following result provides a characterization of exceptional quotient singularities.

Theorem 1.1 ([7, Theorem 3.17]). Let $G \subset \mathrm{GL}_{n+1}(\mathbb{C})$ be a finite subgroup that does not contain reflections. Then $\mathbb{C}^{n+1} / G$ is exceptional if and only if for any $\bar{G}$-invariant effective $\mathbb{Q}$-divisor $D$ on $\mathbb{P}^{n}$ such that $D \sim_{\mathbb{Q}}-K_{\mathbb{P}^{n}}$, the log pair $\left(\mathbb{P}^{n}, D\right)$ is Kawamata log terminal. 
Corollary 1.1. Let $G \subset \mathrm{GL}_{n+1}(\mathbb{C})$ be a finite subgroup that does not contain reflections. Then the singularity $\mathbb{C}^{n+1} / G$ is exceptional if $\operatorname{lct}\left(\mathbb{P}^{n}, \bar{G}\right)>1$. Moreover, the singularity $\mathbb{C}^{n+1} / G$ is not exceptional if either $\operatorname{lct}\left(\mathbb{P}^{n}, \bar{G}\right)<1$, or $G$ has a semiinvariant of degree at most $n+1$.

Note that the assumption that $G$ contains no reflections is crucial for Theorem 1.1.

Example 1.2. Let $G \subset \mathrm{GL}_{4}(\mathbb{C})$ be the subgroup number 32 in [26, Table VII]. Then $G$ is generated by reflections (see $[26]$ ). Thus, the singularity $\mathbb{C}^{4} / G$ is not exceptional by Remark 1.2. On the other hand, it follows from [7, Theorem 4.13] that $\operatorname{lct}\left(\mathbb{P}^{3}, \bar{G}\right) \geqslant 5 / 4$. One can produce similar examples for two-dimensional and three-dimensional singularities.

Definition 1.4 (see [3]). The subgroup $G \subset \mathrm{GL}_{n+1}(\mathbb{C})$ is primitive if there is no nontrivial decomposition

$$
\mathbb{C}^{n+1}=\bigoplus_{i=1}^{r} V_{i}
$$

such that for any $g \in G$ and any $i$ there is some $j=j(g)$ such that $g\left(V_{i}\right)=V_{j}$. The subgroup $\bar{G} \subset \mathrm{PGL}_{n+1}(\mathbb{C})$ is primitive if $G$ is primitive.

Up to conjugation, there are finitely many primitive finite subgroups in $\mathrm{SL}_{n+1}(\mathbb{C})$ (see $[9])$.

Theorem 1.2 (see [24, Proposition 2.1] or [7, Corollary 3.20]). Let $G \subset \mathrm{GL}_{n+1}(\mathbb{C})$ be a finite subgroup that does not contain reflections. If $\mathbb{C}^{n+1} / G$ is exceptional, then $G$ is primitive.

Exceptional quotient singularities of dimension up to 5 are classified in $[7,20,27]$.

Theorem 1.3 ([7, Theorem 1.22], $[20,27])$. Let $G \subset \mathrm{GL}_{n+1}(\mathbb{C})$ be a finite subgroup without reflections. If $n \leqslant 4$, then the following are equivalent:

- the singularity $\mathbb{C}^{n+1} / G$ is exceptional,

- the inequality $\operatorname{lct}\left(\mathbb{P}^{n}, \bar{G}\right) \geqslant(n+2) /(n+1)$ holds,

- the group $G$ is primitive and has no semi-invariants of degree at most $n+1$.

The assertion of Theorem 1.3 is no longer true if $n \geqslant 5$ (see [7, Example 3.25]).

Remark 1.3. Let $G \subset \mathrm{GL}_{2}(\mathbb{C})$ be a finite subgroup. Then

$$
\operatorname{lct}\left(\mathbb{P}^{1}, \bar{G}\right)=\left\{\begin{array}{l}
6 \text { if } \bar{G} \cong \mathrm{A}_{5} \\
4 \text { if } \bar{G} \cong \mathrm{S}_{4} \\
2 \text { if } \bar{G} \cong \mathrm{A}_{4}, \\
1 \text { if } \bar{G} \cong \mathrm{D}_{m} \\
1 / 2 \text { if } \bar{G} \cong \mathbb{Z}_{m} .
\end{array}\right.
$$

The main purpose of this paper is to prove the following result. 
Theorem 1.4. Let $G \subset \mathrm{SL}_{6}(\mathbb{C})$ be a finite subgroup. Then the following are equivalent:

- the singularity $\mathbb{C}^{6} / G$ is exceptional,

- the inequality $\operatorname{lct}\left(\mathbb{P}^{5}, \bar{G}\right) \geqslant 7 / 6$ holds,

- either $\bar{G}$ is the Hall-Janko group HaJ (see $[16,17])$, or $G \cong 6 . \mathrm{A}_{7}$ and $\bar{G} \cong \mathrm{A}_{7}$.

Proof. The required assertion follows from Theorems 3.2, 4.1, 4.2 and Lemma 2.6.

As far as we know, Theorem 1.4 gives the first appearance of the Hall--Janko group HaJ in algebraic geometry. The assertion of Theorem 1.4 gives new examples of normalized Kähler-Ricci iterations that converge to the Fubini-Study metric on $\mathbb{P}^{5}$ (see cf. [7, Question 1.9], [25]). Furthermore, it follows from Corollary 1.1 that Theorem 1.4 can be considered as a classification of six-dimensional exceptional quotient singularities.

Remark 1.4. Suppose that $\bar{G} \subset \mathrm{PGL}_{6}(\mathbb{C})$. If $\bar{G} \cong$ HaJ, then there exist two subgroups in $\mathrm{SL}_{6}(\mathbb{C})$ whose images in $\mathrm{PGL}_{6}(\mathbb{C})$ coincide with $\bar{G}$. Namely, one of them is isomorphic to 2.HaJ, and another one is isomorphic to the extension of the subgroup 2.HaJ $\subset \mathrm{SL}_{6}(\mathbb{C})$ by a scalar matrix with non-zero entries equal to a primitive root of unity of degree 6 . Moreover, up to conjugation $\mathrm{PGL}_{6}(\mathbb{C})$ contains a unique subgroup isomorphic to HaJ. On the other hand, $\mathrm{PGL}_{6}(\mathbb{C})$ contains non-conjugate subgroups isomorphic to $\mathbb{A}_{7}$. Furthermore, if $\bar{G} \cong \mathbb{A}_{7}$ and the singularity $\mathbb{C}^{6} / G$ is exceptional, then we must necessarily have $G \cong 6 . \mathrm{A}_{7}$, which uniquely determines the subgroup $\bar{G} \subset \mathrm{PGL}_{6}(\mathbb{C})$ up to conjugation. Other alternatives for a minimal lift $G$ of a primitive group $\mathrm{A}_{7} \cong \bar{G} \subset \mathrm{PGL}_{6}(\mathbb{C})$ are $G \cong 3 . \mathrm{A}_{7}$ and $G \cong \mathrm{A}_{7}$ (see Theorem 3.1), which happens for two other classes of subgroups $\mathrm{A}_{7} \cong \bar{G} \subset \mathrm{PGL}_{6}(\mathbb{C})$. In the latter cases the singularity $\mathbb{C}^{6} / G$ is not exceptional.

Finally, we prove the following surprising result.

Theorem 1.5 (cf. [7, Example 3.13]). There are no exceptional quotient singularities of dimension 7 .

The plan of the paper is as follows. In Section 2, we collect well-known auxiliary results. In Section 3, we show that apart from the singularities related to the groups $6 . \mathrm{A}_{7}$ and 2.HaJ all other six-dimensional quotient singularities are not exceptional. In Section 4, we prove the exceptionality of the singularities related to the groups $6 . \mathrm{A}_{7}$ and 2.HaJ thus completing the proof of Theorem 1.4. Finally, in Section 5 we prove Theorem 1.5. In Appendix A, we introduce a new invariant of a Kawamata $\log$ terminal singularity based on the classical $\alpha$-invariant of Tian.

Throughout the paper we use usual notation for cyclic, dihedral, symmetric and alternating groups, as well as for standard algebraic groups. For a group $\Gamma$ we denote by $k . \Gamma$ a (non-trivial) central extension of $\Gamma$ by the central subgroup $\mathbb{Z}_{k}$ (this might be non-unique).

Many of the computations with the characters of large finite groups we need are too complicated to make by hand (namely, those mentioned in the proofs of Theorem 3.2, 4.1 and 4.2 and in Remark 3.1). In such cases we used the Magma software [2]. 


\section{Preliminaries}

Let $X$ be a variety with at most Kawamata log terminal singularities (see [13, Definition 3.5]), let $B_{X}$ be an effective $\mathbb{Q}$-divisor on the variety $X$ such that $\left(X, B_{X}\right)$ is log canonical. Then

$$
B_{X}=\sum_{i=1}^{r} a_{i} B_{i}
$$

where $a_{i} \in \mathbb{Q}_{\geqslant 0}$, and $B_{i}$ is a prime Weil divisor on the variety $X$.

Let $\pi: \bar{X} \rightarrow X$ be a birational morphism such that $\bar{X}$ is smooth. Then

$$
K_{\bar{X}}+\sum_{i=1}^{r} a_{i} \bar{B}_{i} \sim_{\mathbb{Q}} \pi^{*}\left(K_{X}+B_{X}\right)+\sum_{i=1}^{m} d_{i} E_{i},
$$

where $\bar{B}_{i}$ is the proper transforms of the divisor $B_{i}$ on the variety $\bar{X}$, and $E_{i}$ is an exceptional divisor of the morphism $\pi$, and $d_{i}$ is a rational number. We may assume that

$$
\left(\bigcup_{i=1}^{r} \bar{B}_{i}\right) \bigcup\left(\bigcup_{i=1}^{m} E_{i}\right)
$$

is a divisor with simple normal crossing. Put

$$
\mathcal{I}\left(X, B_{X}\right)=\pi_{*} \mathcal{O}_{\bar{X}}\left(\sum_{i=1}^{m}\left\lceil d_{i}\right\rceil E_{i}-\sum_{i=1}^{r}\left\lfloor a_{i}\right\rfloor B_{i}\right) .
$$

Theorem 2.1 ([15, Theorem 9.4.8]). Let $H$ be a nef and big $\mathbb{Q}$-divisor on $X$ such that

$$
K_{X}+B_{X}+H \equiv D
$$

where $D$ is a Cartier divisor on the variety $X$. Then $H^{i}\left(\mathcal{I}\left(X, B_{X}\right) \otimes D\right)=0$ for every $i \geqslant 1$.

Let $\mathcal{L}\left(X, B_{X}\right)$ be a subscheme that corresponds to the ideal sheaf $\mathcal{I}\left(X, B_{X}\right)$. Put

$$
\operatorname{LCS}\left(X, B_{X}\right)=\operatorname{Supp}\left(\mathcal{L}\left(X, B_{X}\right)\right) .
$$

The subscheme $\mathcal{L}\left(X, B_{X}\right)$ is reduced, because $\left(X, B_{X}\right)$ is log canonical. Note that

- $\mathcal{I}\left(X, B_{X}\right)$ is known as the multiplier ideal sheaf (see [15, Section 9.2]),

- $\mathcal{L}\left(X, B_{X}\right)$ is known as the log canonical singularities subscheme (see [6, Definition 2.5]),

- $\operatorname{LCS}\left(X, B_{X}\right)$ is known as the locus of log canonical singularities (see [27, Definition 3.14]).

Let $Z$ be a center of log canonical singularities of the log pair $\left(X, B_{X}\right)$ (see [11, Definition 1.3]), and let $\mathbb{L} \mathbb{C} \mathbb{S}\left(X, B_{X}\right)$ be the set of all centers of $\log$ canonical singularities of the log pair $\left(X, B_{X}\right)$.

Lemma 2.1 ([11, Proposition 1.5]). Let $Z^{\prime}$ be an element of the set $\mathbb{L} \mathbb{C} S\left(X, B_{X}\right)$ such that

$$
\varnothing \neq Z \cap Z^{\prime}=\sum_{i=1}^{k} Z_{i}
$$


where $Z_{i} \subsetneq Z$ is an irreducible subvariety. Then $Z_{i} \in \mathbb{L} \mathbb{C} S\left(X, B_{X}\right)$ for every $i \in\{1, \ldots, k\}$.

Suppose that $Z$ is a minimal center in $\mathbb{L} \mathbb{C} S\left(X, B_{X}\right)$ (see $\left.[11,12]\right)$.

Theorem 2.2 ([12, Theorem 1$])$. The variety $Z$ is normal and has at most rational singularities. For every ample $\mathbb{Q}$-Cartier $\mathbb{Q}$-divisor $\Delta$ on $X$ there exists an effective $\mathbb{Q}$-divisor $B_{Z}$ on the variety $Z$ such that

$$
\left.\left(K_{X}+B_{X}+\Delta\right)\right|_{Z} \sim_{\mathbb{Q}} K_{Z}+B_{Z}
$$

and $\left(Z, B_{Z}\right)$ has Kawamata log terminal singularities.

Remark 2.1. In the notation and assumptions of Theorem 2.2, suppose that

$$
K_{X}+B_{X}+\Delta \sim_{\mathbb{Q}} D
$$

where $D$ is a Cartier divisor on $X$. Put $H=\left.D\right|_{Z}$. Let $\nu: \bar{Z} \rightarrow Z$ be a desingularization. Then

$$
h^{0}\left(\mathcal{O}_{Z}(H)\right)=\chi\left(\mathcal{O}_{Z}(H)\right)=\chi\left(\mathcal{O}_{\bar{Z}}\left(\nu^{*}(H)\right)\right)
$$

by Theorem 2.1, because $Z$ has at most rational singularities by Theorem 2.2.

Let $\bar{G} \subseteq \operatorname{Aut}(X)$ be a finite subgroup. Suppose that $B_{X}$ is $\bar{G}$-invariant. Then $g(Z) \in \mathbb{L} \mathbb{C S}\left(X, B_{X}\right)$ for every $g \in \bar{G}$, and the locus $\operatorname{LCS}\left(X, B_{X}\right)$ is $\bar{G}$-invariant. It follows from Lemma 2.1 that

$$
g(Z) \cap g^{\prime}(Z) \neq \varnothing \Longleftrightarrow g(Z)=g^{\prime}(Z)
$$

for every $g \in \bar{G} \ni g^{\prime}$, because $Z$ is a minimal center in $\mathbb{L} \mathbb{C} \mathbb{S}\left(X, B_{X}\right)$.

Lemma 2.2. Suppose that the divisor $B_{X}$ is ample. Let $\epsilon$ be an arbitrary rational number such that $\epsilon>1$. Then there exists an effective $\bar{G}$-invariant $\mathbb{Q}$-divisor $D$ on the variety $X$ such that

$$
\mathbb{L} \mathbb{C S}(X, D)=\bigcup_{g \in \bar{G}}\{g(Z)\},
$$

the log pair $(X, D)$ is log canonical, and the equivalence $D \sim_{\mathbb{Q}} \epsilon\left(B_{X}\right)$ holds.

Proof. See the proofs of [11, Theorem 1.10], [12, Theorem 1], [7, Lemma 2.8].

Suppose that $X \cong \mathbb{P}^{n}$. Let $H$ be a hyperplane in $\mathbb{P}^{n}$. Suppose that

$$
\mathbb{L} \mathbb{C} S\left(X, B_{X}\right)=\bigcup_{g \in \bar{G}}\{g(Z)\},
$$

and let $Y$ be the $\bar{G}$-orbit of the subvariety $Z \subset \mathbb{P}^{n}$.

Lemma 2.3. Put $s=n-\operatorname{dim}(Y)$ and

$$
r=\left\{\begin{array}{l}
\lceil\mu-s-1\rceil+1, \quad \text { if } \mu \in \mathbb{Z}, \\
\lceil\mu-s-1\rceil, \quad \text { if } \mu \notin \mathbb{Z},
\end{array}\right.
$$

where $\mu \in \mathbb{Q}$ such that $B_{X} \sim_{\mathbb{Q}} \mu H$. Then $r \geqslant 0$ and

$$
\operatorname{deg}(Y) \leqslant\left(\begin{array}{c}
s+r \\
r
\end{array}\right)
$$


Proof. Let $\Pi \subset \mathbb{P}^{n}$ be a general linear subspace of dimension $s$. Put

$$
D=\left.B_{X}\right|_{\Pi}
$$

and $\Lambda=H \cap \Pi$. Then $\operatorname{deg}(Y)=|Y \cap \Pi|$ and $\operatorname{LCS}(\Pi, D)=Y \cap \Pi$. One has

$$
K_{\Pi}+D \sim_{\mathbb{Q}}(\mu-s-1) \Lambda .
$$

It follows from Theorem 2.1 that there is an exact sequence of cohomology groups

$$
0 \longrightarrow H^{0}\left(\mathcal{O}_{\Pi}(r \Lambda) \otimes \mathcal{I}(\Pi, D)\right) \longrightarrow H^{0}\left(\mathcal{O}_{\Pi}(r \Lambda)\right) \longrightarrow H^{0}\left(\mathcal{O}_{\mathcal{L}(\Pi, D)}\right) \longrightarrow 0,
$$

and $\operatorname{Supp}(\mathcal{L}(\Pi, D))=\operatorname{LCS}(\Pi, D)=Y \cap \Pi \neq \varnothing$. Therefore, we see that $r \geqslant 0$ and

$$
\operatorname{deg}(Y)=|Y \cap \Pi| \leqslant h^{0}\left(\mathcal{O}_{\mathcal{L}(\Pi, D)}\right) \leqslant h^{0}\left(\mathcal{O}_{\Pi}(r \Lambda)\right)=h^{0}\left(\mathcal{O}_{\mathbb{P}}(r)\right)=\left(\begin{array}{c}
s+r \\
r
\end{array}\right)
$$

which completes the proof.

Let $G$ be a finite subgroup in $\mathrm{GL}_{n+1}(\mathbb{C})$ such that $\bar{G}=\phi(G)$, where the map $\phi: \mathrm{GL}_{n+1}(\mathbb{C}) \rightarrow \operatorname{Aut}\left(\mathbb{P}^{n}\right) \cong \mathrm{PGL}_{n+1}(\mathbb{C})$ is the natural projection.

Lemma 2.4. If $G$ is conjugate to a subgroup in $\mathrm{GL}_{n+1}(\mathbb{R})$, then $G$ has an invariant of degree 2 .

Proof. If $G$ is conjugate to a subgroup in $\mathrm{GL}_{n+1}(\mathbb{R})$, then there exists a (real positive definite) $G$-invariant inner product, which gives a non-trivial $G$-invariant element in $\operatorname{Sym}^{2}\left(\mathbb{C}^{n+1}\right)$.

Lemma 2.5. Suppose that there exists a normal subgroup $F \subset G$ such that $G / F$ is an abelian group, and $F$ has an invariant of degree $d$. Then $G$ has a semi-invariant of degree $d$.

Proof. Let $V$ be a space of invariants of the group $F$ of degree $d$. Then the group $G / F$ naturally acts on the space $V$. Since the group $G / F$ is abelian, it has a onedimensional invariant subspace, which gives a required semi-invariant of the subgroup $G$.

Let $G_{1} \subset \mathrm{SL}_{2}(\mathbb{C})$ and $G_{2} \subset \mathrm{SL}_{l}(\mathbb{C})$ be finite subgroups, let $\mathbb{M}$ be the vector space of $2 \times l$-matrices with entries in $\mathbb{C}$. For every $\left(g_{1}, g_{2}\right) \in G_{1} \times G_{2}$ and every $M \in \mathbb{M}$, put

$$
\left(g_{1}, g_{2}\right)(M)=g_{1} M g_{2}^{-1} \in \mathbb{M} \cong \mathbb{C}^{2 l},
$$

which induces a homomorphism $\varphi: G_{1} \times G_{2} \rightarrow \mathrm{SL}_{2 l}(\mathbb{C})$. Note that $|\operatorname{ker}(\varphi)| \leqslant 2$ if $n$ is even, and $\varphi$ is a monomorphism if $n$ is odd. Suppose that $n=2 l-1 \geqslant 3$.

Lemma 2.6 ([7, Lemma 3.24]). Suppose that $G=\varphi\left(G_{1} \times G_{2}\right)$. Then $\operatorname{lct}\left(\mathbb{P}^{n}, \bar{G}\right)<1$. Proof. Put $s=l-1$. Let $\psi: \mathbb{P}^{1} \times \mathbb{P}^{s} \rightarrow \mathbb{P}^{n}$ be the Segre embedding. Put $Y=$ $\psi\left(\mathbb{P}^{1} \times \mathbb{P}^{s}\right)$ and let $\mathcal{Q}$ be the linear system consisting of all quadric hypersurfaces in $\mathbb{P}^{n}$ that pass through the subvariety $Y$. Then $\mathcal{Q}$ is a non-empty $\bar{G}$-invariant linear system. The log pair $\left(\mathbb{P}^{n}, l \mathcal{Q}\right)$ is not log-canonical along $Y$. Now it follows from $[13$, Theorem 4.8] that $\operatorname{lct}\left(\mathbb{P}^{n}, \bar{G}\right)<1$. 


\section{Six-dimensional case}

Let $G$ be a finite subgroup in $\mathrm{SL}_{n+1}(\mathbb{C})$. Put $V=\mathbb{C}^{n+1}$.

Definition 3.1 (see [19, Section 1]). The subgroup $G$ is quasiprimitive if the following conditions hold:

- the vector space $V$ is an irreducible representation of the group $G$,

- for any nontrivial normal subgroup $N \subseteq G$ one has $V \cong W^{\oplus r}$ as a representation of $N$, where $W$ is an irreducible representation of $N$, and $r \geqslant 1$.

Suppose that $n=5$. Let $\phi: \mathrm{SL}_{6}(\mathbb{C}) \rightarrow \operatorname{Aut}\left(\mathbb{P}^{5}\right)$ be the natural projection. Put $\bar{G}=\phi(G)$. We say that the subgroup $G$ is the lift of the subgroup $\bar{G} \subset \operatorname{Aut}\left(\mathbb{P}^{5}\right) \cong$ $\mathrm{PGL}_{6}(\mathbb{C})$ to $\mathrm{SL}_{6}(\mathbb{C})$.

Theorem 3.1 ([18, Section 3]). Suppose that $G$ is quasiprimitive. Then there exists a lift of the subgroup $\bar{G} \subset \operatorname{Aut}\left(\mathbb{P}^{5}\right)$ to $\mathrm{SL}_{6}(\mathbb{C})$ that is contained in the following list:

(I) (i) a subgroup of the group $\mathrm{SL}_{6}(\mathbb{C})$ satisfying the hypotheses of Lemma 2.6,

(ii) a certain subgroup of a subgroup described in $I(i)$ (see $[18, \S 3]$ for details),

(II) $\mathrm{SL}_{2}\left(\mathbb{F}_{5}\right)$,

(III) $2 . \mathrm{S}_{5}$,

(IV) (i) $3 . A_{6}$,

(ii) an extension of the subgroup described in IV(i) by an automorphism of order 2 ,

(V) $6 . \mathrm{A}_{6}$,

(VI) $\mathrm{A}_{7}$ or $\mathrm{S}_{7}$,

(VII) $3 . \mathrm{A}_{7}$,

(VIII) $6 . \mathrm{A}_{7}$,

(IX) (i) $\mathrm{PSL}_{2}\left(\mathbb{F}_{7}\right)$,

(ii) $\mathrm{PGL}_{2}\left(\mathbb{F}_{7}\right)$,

(X) (i) $\mathrm{SL}_{2}\left(\mathbb{F}_{7}\right)$,

(ii) an extension of the subgroup described in $X(i)$ by an automorphism of order 2 ,

(XI) $\mathrm{SL}_{2}\left(\mathbb{F}_{11}\right)$,

(XII) $\mathrm{SL}_{2}\left(\mathbb{F}_{13}\right)$,

(XIII) (i) $\mathrm{PSp}_{4}\left(\mathbb{F}_{3}\right)$,

(ii) an extension of the subgroup described in XIII(i) by an automorphism of order 2 ,

(XIV) (i) $\mathrm{SU}_{3}\left(\mathbb{F}_{3}\right)$,

(ii) an extension of the subgroup described in XIV(i) by an automorphism of order 2 ,

$(\mathrm{XV}) \quad$ (i) $6 . \mathrm{PSU}_{4}\left(\mathbb{F}_{3}\right)$,

(ii) an extension of the subgroup described in $X V(i)$ by an automorphism of order 2 ,

(XVI) 2.HaJ, where HaJ is the Hall-Janko group (see [16], [17]),

(XVII) (i) $6 . \mathrm{PSL}_{3}\left(\mathbb{F}_{4}\right)$,

(ii) an extension of the subgroup described in XVII(i) by an automorphism of order 2 .

Recall that all primitive subgroups are quasiprimitive (see [19, §1]). 
The main purpose of this section is to prove the following result.

Theorem 3.2. If $G$ is primitive, then $G$ has a semi-invariant of degree at most 6 unless there exists a lift of $\bar{G}$ to $\mathrm{SL}_{6}(\mathbb{C})$ that is a group of type I, VIII or XVI in the notation of Theorem 3.1.

Proof. Recall that changing a lift of $\bar{G}$ to $\mathrm{SL}_{6}(\mathbb{C})$ does not change the degrees of semi-invariants, which implies that we may assume that $G$ is one of the groups listed in Theorem 3.1.

If the subgroup $G$ is of type VI, IX(i) or XIII(i), then the subgroup $G$ is conjugate to a subgroup of $\mathrm{SL}_{6}(\mathbb{Q})$ (see $[10]$ ), and hence $G$ has an invariant of degree 2 by Lemma 2.4.

If the subgroup $G$ is of type $\mathrm{XV}(\mathrm{i})$, then $G$ is a subgroup of the Mitchell group $6 . \mathrm{PSU}_{4}\left(\mathbb{F}_{3}\right) .2$, which is the group number 34 in [26, Table VII], and hence the subgroup $G$ has an invariant of degree 6 , because the Mitchell group has an invariant of degree 6 (see [26, Table VII]).

If the subgroup $G$ is of type II, V, VII, X(i), XI, XII, XIV(i) or XVII(i), then the minimal degree $d_{\min }$ of the invariants of the subgroup $G$ is given in the following table:

\begin{tabular}{|c|c|c|c|c|c|c|c|c|}
\hline$G$ & $2 . \mathrm{A}_{5}$ & $6 . \mathrm{A}_{6}$ & $3 . \mathrm{A}_{7}$ & $\mathrm{SL}_{2}\left(\mathbb{F}_{7}\right)$ & $\mathrm{SL}_{2}\left(\mathbb{F}_{11}\right)$ & $\mathrm{SL}_{2}\left(\mathbb{F}_{13}\right)$ & $\mathrm{SU}_{3}\left(\mathbb{F}_{3}\right)$ & $6 . \mathrm{PSL}_{3}\left(\mathbb{F}_{4}\right)$ \\
\hline Type & $\mathrm{II}$ & $\mathrm{V}$ & $\mathrm{VII}$ & $\mathrm{X}(\mathrm{i})$ & $\mathrm{XI}$ & $\mathrm{XII}$ & $\mathrm{XIV}(\mathrm{i})$ & $\mathrm{XVII}(\mathrm{i})$ \\
\hline$d_{\text {min }}$ & 4 & 6 & 3 & 4 & 4 & 4 & 6 & 6 \\
\hline
\end{tabular}

If the subgroup $G$ is a subgroup of type $\operatorname{IV}(\mathrm{i})$, then $G$ is a subgroup of a quasiprimitive subgroup of type VII, which implies that the subgroup $G$ has an invariant of degree 3 .

If the subgroup $G$ is a subgroup of type III, then it has a normal subgroup isomorphic to $2 . \mathrm{A}_{5}$, which implies that $G$ has a semi-invariant of degree 4 by Lemma 2.5 .

Arguing as in the case of a subgroup of type III, we see that the subgroup $G$ has a semi-invariant of degree $3,2,4,2,6,6$ or 6 in the case when the subgroup $G \subset \mathrm{SL}_{6}(\mathbb{C})$ is a quasiprimitive subgroup of type $\mathrm{IV}(\mathrm{ii}), \mathrm{IX}(\mathrm{ii}), \mathrm{X}(\mathrm{ii}), \mathrm{XIII}(\mathrm{ii}), \mathrm{XIV}(\mathrm{ii})$, $\mathrm{XV}(\mathrm{ii})$ or XVII(ii), respectively.

Remark 3.1. In the notation of Theorem 3.1, if $G$ is a primitive subgroup of type VIII or XVI, then a direct computation shows that the minimal degree of the semiinvariants of $G$ equals 12 .

\section{Exceptional cases}

Let $G$ be a subgroup in $\mathrm{SL}_{6}(\mathbb{C})$. Define $V$ and $\bar{G}$ as in Section 3 .

Remark 4.1. If the group $\bar{G}$ is a simple non-abelian group such that $Z(G) \subseteq[G, G]$, where $Z(G)$ and $[G, G]$ denote the center and the commutator of the subgroup $G$, respectively, then every semi-invariant of the group $G$ is its invariant.

Theorem 4.1. Suppose that $\bar{G} \cong$ HaJ is the Hall-Janko group. Then one has $\operatorname{lct}\left(\mathbb{P}^{5}, \bar{G}\right) \geqslant 7 / 6$. 
Proof. We may assume that $G \cong 2$.HaJ (see Theorem 3.1). Then $Z(G) \subseteq[G, G]$.

Suppose that $\operatorname{lct}\left(\mathbb{P}^{5}, \bar{G}\right)<7 / 6$. Then there is an effective $\bar{G}$-invariant $\mathbb{Q}$-divisor

$$
D \sim_{\mathbb{Q}}-K_{\mathbb{P}^{5}} \sim \mathcal{O}_{\mathbb{P}^{5}}(6),
$$

and there is a positive rational number $\lambda<7 / 6$ such that $\left(\mathbb{P}^{5}, \lambda D\right)$ is strictly log canonical.

Let $S$ be a minimal center in $\mathbb{L} \mathbb{C S}\left(\mathbb{P}^{5}, \lambda D\right)$, let $Z$ be the $\bar{G}$-orbit of the subvariety $S \subset \mathbb{P}^{5}$, and let $r$ be the number of irreducible components of the subvariety $Z$. We may assume that

$$
\mathbb{L} \mathbb{C S}\left(\mathbb{P}^{5}, \lambda D\right)=\bigcup_{g \in \bar{G}}\{g(S)\}
$$

by Lemma 2.2. Then $\operatorname{Supp}(Z)=\operatorname{LCS}\left(\mathbb{P}^{5}, \lambda D\right)$. It follows from Lemma 2.1 that:

$$
g(S) \cap g^{\prime}(S) \neq \varnothing \Longleftrightarrow g(S)=g^{\prime}(S),
$$

for every $g \in \bar{G} \ni g^{\prime}$. Then $\operatorname{deg}(Z)=r \operatorname{deg}(S)$.

It follows from Remark 3.1 that the subgroup $G$ does not have invariants of degree up to 6 , which immediately implies that $\operatorname{dim}(S) \neq 4$ by Remark 4.1 .

Let $\mathcal{I}$ be the multiplier ideal sheaf of the $\log$ pair $\left(\mathbb{P}^{5}, \lambda D\right)$, and let $\mathcal{L}$ be the log canonical singularities subscheme of the $\log$ pair $\left(\mathbb{P}^{5}, \lambda D\right)$. By Theorem 2.1, there is an exact sequence

$$
0 \longrightarrow H^{0}\left(\mathcal{O}_{\mathbb{P}^{5}}(n) \otimes \mathcal{I}\right) \longrightarrow H^{0}\left(\mathcal{O}_{\mathbb{P}^{5}}(n)\right) \longrightarrow H^{0}\left(\mathcal{O}_{\mathcal{L}} \otimes \mathcal{O}_{\mathbb{P}^{5}}(n)\right) \longrightarrow 0,
$$

for every $n \geqslant 1$. A direct computation shows that $\operatorname{Sym}^{n}(V)$ is an irreducible representation of the group $G$ for all $n \leqslant 5$. Hence, we see that

$$
h^{0}\left(\mathcal{O}_{\mathbb{P}^{5}}(n) \otimes \mathcal{I}\right)=0,
$$

for every $n \in\{1,2,3,4,5\}$. Note that $Z=\mathcal{L}$, because $\left(\mathbb{P}^{5}, \lambda D\right)$ is $\log$ canonical. Thus, we have

$$
h^{0}\left(\mathcal{O}_{Z} \otimes \mathcal{O}_{\mathbb{P}^{5}}(n)\right)=h^{0}\left(\mathcal{O}_{\mathbb{P}^{5}}(n)\right)=\left(\begin{array}{c}
5+n \\
n
\end{array}\right),
$$

for every $n \in\{1,2,3,4,5\}$. In particular, we see that $r \leqslant 6$, because

$$
r h^{0}\left(\mathcal{O}_{S} \otimes \mathcal{O}_{\mathbb{P}^{5}}(1)\right)=h^{0}\left(\mathcal{O}_{Z} \otimes \mathcal{O}_{\mathbb{P}^{5}}(1)\right)=6,
$$

which implies that $r=1$, because $\bar{G}$ has no nontrivial maps to $\mathrm{S}_{r}$ for $2 \leqslant r \leqslant 6$.

Note that it follows from the equality $r=1$ that $\operatorname{dim}(S) \neq 0$.

Let $H$ be a hyperplane section of the variety $S \subset \mathbb{P}^{5}$. It follows from Theorem 2.2 that the variety $S$ is normal and has at most rational singularities, and there are an effective $\mathbb{Q}$-divisor $B_{S}$ and an ample $\mathbb{Q}$-divisor $\Delta$ on the surface $S$ such that $K_{S}+B_{S}+\Delta \sim_{\mathbb{Q}} H$, and the log pair $\left(S, B_{S}\right)$ has Kawamata log terminal singularities.

Using the Riemann-Roch theorem and Remark 2.1, we see that $\chi\left(\mathcal{O}_{S}(n H)\right)$ is a polynomial in $n$ of degree at most $\operatorname{dim}(S)$ such that

$$
\chi\left(\mathcal{O}_{S}(n H)\right)=h^{0}\left(\mathcal{O}_{S}(n H)\right),
$$


for any $n \geqslant 1$. On the other hand, it follows from (4.1) that:

$$
\chi\left(\mathcal{O}_{S}(n H)\right)= \begin{cases}6 & \text { if } n=1, \\ 21 & \text { if } n=2, \\ 56 & \text { if } n=3, \\ 126 & \text { if } n=4, \\ 252 & \text { if } n=5,\end{cases}
$$

which gives an inconsistent system of linear equations on the coefficients of the polynomial $\chi\left(\mathcal{O}_{S}(n H)\right)$, since $\operatorname{dim}(S) \leqslant 3$.

Theorem 4.2. Suppose that $G \cong 6 \cdot \mathrm{A}_{7}$. Then $\operatorname{lct}\left(\mathbb{P}^{5}, \bar{G}\right) \geqslant 7 / 6$.

Proof. Suppose that $\operatorname{lct}\left(\mathbb{P}^{5}, \bar{G}\right)<7 / 6$. Then there is an effective $\bar{G}$-invariant $\mathbb{Q}$-divisor

$$
D \sim{ }_{\mathbb{Q}}-K_{\mathbb{P}^{5}} \sim \mathcal{O}_{\mathbb{P}^{5}}(6),
$$

and there is a positive rational number $\lambda<7 / 6$ such that $\left(\mathbb{P}^{5}, \lambda D\right)$ is strictly $\log$ canonical.

Arguing as in the proof of Theorem 4.1, we may assume that

$$
\mathbb{L} \mathbb{C S}\left(\mathbb{P}^{5}, \lambda D\right)=\bigcup_{g \in \bar{G}}\{g(S)\},
$$

where $S$ is a minimal center of $\log$ canonical singularities of the log pair $\left(\mathbb{P}^{5}, \lambda D\right)$.

Let $Z$ be the $\bar{G}$-orbit of the subvariety $S \subset \mathbb{P}^{5}$. Then $\operatorname{LCS}\left(\mathbb{P}^{5}, \lambda D\right)=\operatorname{Supp}(Z)$.

It follows from Remark 3.1 that the subgroup $G$ does not have invariants of degree up to 6 , which immediately implies that $\operatorname{dim}(S) \neq 4$ by Remark 4.1.

Let $\mathcal{I}$ be the multiplier ideal sheaf of the $\log$ pair $\left(\mathbb{P}^{5}, \lambda D\right)$, and let $\mathcal{L}$ be the $\log$ canonical singularities subscheme of the $\log$ pair $\left(\mathbb{P}^{5}, \lambda D\right)$. By Theorem 2.1, we have

$$
\chi\left(\mathcal{O}_{Z}(n H)\right)=h^{0}\left(\mathcal{O}_{Z}(n H)\right)=\left(\begin{array}{c}
5+n \\
n
\end{array}\right)-h^{0}\left(\mathcal{O}_{\mathbb{P}^{5}}(n) \otimes \mathcal{I}\right),
$$

for every $n \geqslant 1$, because $Z=\mathcal{L}$. Put $q_{n}=h^{0}\left(\mathcal{O}_{\mathbb{P}^{5}}(n) \otimes \mathcal{I}\right)$ for every $n \geqslant 1$. Then

$$
q_{1}=q_{2}=0,
$$

because $V$ and $\operatorname{Sym}^{2}(V)$ are irreducible representations of the group $G$. Hence

$$
h^{0}\left(\mathcal{O}_{S} \otimes \mathcal{O}_{\mathbb{P}^{5}}(n)\right)=h^{0}\left(\mathcal{O}_{\mathbb{P}^{5}}(n)\right)=\left(\begin{array}{c}
5+n \\
n
\end{array}\right)
$$

for $n \in\{1,2\}$ by (4.2). In particular, we see that $r \leqslant 6$, because

$$
r h^{0}\left(\mathcal{O}_{S} \otimes \mathcal{O}_{\mathbb{P}^{5}}(1)\right)=h^{0}\left(\mathcal{O}_{Z} \otimes \mathcal{O}_{\mathbb{P}^{5}}(1)\right)=6
$$

which implies that $r=1$ and $Z=S$, because $\bar{G}$ has no nontrivial maps to $\mathrm{S}_{r}$ for $2 \leqslant r \leqslant 6$.

Note that it follows from the equality $r=1$ that $\operatorname{dim}(S) \neq 0$.

Similarly, we see that $q_{3} \in\{0,20,36\}$, because

$$
\operatorname{Sym}^{3}(V)=T_{36} \oplus T_{20},
$$


where $T_{i}$ is an irreducible representation of the group $G$ of dimension $i$. Thus, we have

$$
\begin{aligned}
h^{0}\left(\mathcal{O}_{S} \otimes \mathcal{O}_{\mathbb{P}^{5}}(3)\right) & =h^{0}\left(\mathcal{O}_{\mathbb{P}^{5}}(3)\right)-h^{0}\left(\mathcal{O}_{\mathbb{P}^{5}}(3) \otimes \mathcal{I}\right) \\
& =56-h^{0}\left(\mathcal{O}_{\mathbb{P}^{5}}(3) \otimes \mathcal{I}\right) \in\{20,36,56\}
\end{aligned}
$$

by (4.2). Moreover, one has

$$
\operatorname{Sym}^{4}(V)=U_{6} \oplus U_{15} \oplus \hat{U}_{15} \oplus U_{21}^{\oplus 2} \oplus U_{24} \oplus \hat{U}_{24},
$$

where $U_{i}$ and $\hat{U}_{i}$ are irreducible representations of the group $G$ of dimension $i$. In particular,

$$
h^{0}\left(\mathcal{O}_{S} \otimes \mathcal{O}_{\mathbb{P}^{5}}(4)\right)=126-h^{0}\left(\mathcal{O}_{\mathbb{P}^{5}}(4) \otimes \mathcal{I}\right) \notin\{106,114, \ldots, 119,121, \ldots, 125\}
$$

by (4.2). Finally, one has

$$
\operatorname{Sym}^{5}(V)=W_{11}^{\oplus 2} \oplus W_{24}^{\oplus 2} \oplus \hat{W}_{24}^{\oplus 2} \oplus W_{36}^{\oplus 4},
$$

where $W_{i}$ and $\hat{W}_{i}$ are irreducible representations of the group $G$ of dimension $i$. By (4.2), we have

$$
h^{0}\left(\mathcal{O}_{S} \otimes \mathcal{O}_{\mathbb{P}^{5}}(5)\right)=252-h^{0}\left(\mathcal{O}_{\mathbb{P}^{5}}(5) \otimes \mathcal{I}\right) \notin\{66,171,179\} .
$$

Let $H$ be a hyperplane section of the variety $S \subset \mathbb{P}^{5}$. It follows from Theorem 2.2 that the variety $S$ is normal and has at most rational singularities, and there are an effective $\mathbb{Q}$-divisor $B_{S}$ and an ample $\mathbb{Q}$-divisor $\Delta$ on the surface $S$ such that $K_{S}+B_{S}+\Delta \sim_{\mathbb{Q}} H$, and the log pair $\left(S, B_{S}\right)$ has Kawamata log terminal singularities.

Suppose that $\operatorname{dim}(S)=1$. Then $S$ is a smooth curve of genus $g$ such that

$$
\operatorname{deg}(H)=\operatorname{deg}(S)>2 g-2
$$

and $\operatorname{deg}(Z) \leqslant 15$ by Lemma 2.3. By the Riemann-Roch theorem, we get

$$
h^{0}\left(\mathcal{O}_{S}(n H)\right)=n \operatorname{deg}(S)-g+1,
$$

for every $n \geqslant 1$ (see Remark 2.1). Using (4.3), we see that

$$
\left\{\begin{array}{l}
6=\operatorname{deg}(S)-g+1 \\
21=2 \operatorname{deg}(S)-g+1
\end{array}\right.
$$

which implies that $\operatorname{deg}(S)=15$ and $g=10$. Using (4.3) again one obtains

$$
5 \operatorname{deg}(S)-g+1=66
$$

which is impossible by (4.6).

Suppose that $\operatorname{dim}(S)=2$. Using the Riemann-Roch theorem and Remark 2.1, we have

$$
h^{0}\left(\mathcal{O}_{S}(n H)\right)=\chi\left(\mathcal{O}_{S}(n H)\right)=\frac{n^{2}}{2}(H \cdot H)-\frac{n}{2}\left(H \cdot K_{S}\right)+\chi\left(\mathcal{O}_{S}\right)
$$

for any $n \geqslant 1$. Thus, using (4.3) and (4.4), we see that

$$
\left(\operatorname{deg}(S), H \cdot K_{S}, \chi\left(\mathcal{O}_{S}\right)\right) \in\{(5,-5,6),(15,5,-4)\}
$$


because $H \cdot H=\operatorname{deg}(S)>0$. If $\left(\operatorname{deg}(S), H \cdot K_{S}, \chi\left(\mathcal{O}_{S}\right)\right)=(15,5,-4)$, then

$$
h^{0}\left(\mathcal{O}_{S}(4 H)\right)=8(H \cdot H)-2\left(H \cdot K_{S}\right)+\chi\left(\mathcal{O}_{S}\right)=106,
$$

which is impossible by (4.5). If $\left(\operatorname{deg}(S), H \cdot K_{S}, \chi\left(\mathcal{O}_{S}\right)\right)=(15,5,6)$, then

$$
h^{0}\left(\mathcal{O}_{S}(4 H)\right)=116
$$

which is again impossible by (4.5).

We see that $\operatorname{dim}(S)=3$. Then $H \cdot H \cdot H=\operatorname{deg}(S) \geqslant \operatorname{codim}(S)+1=3$, since $V$ is an irreducible representation of the group $G$.

Let $H^{\prime}$ be another general hyperplane section of $S \subset \mathbb{P}^{5}$. Put $C=H \cap H^{\prime}$. Then

$$
-2 \leqslant 2 g(C)-2=H \cdot H \cdot K_{S}+2(H \cdot H \cdot H),
$$

where $g(C)$ is the genus of the curve $C$. Thus, we see that

$$
H \cdot H \cdot K_{S} \geqslant-2-2 \operatorname{deg}(S) \text {. }
$$

By the Riemann-Roch theorem and Remark 2.1, there is $\gamma \in \mathbb{Z}$ such that

$$
h^{0}\left(\mathcal{O}_{S}(n H)\right)=\chi\left(\mathcal{O}_{S}(n H)\right)=\frac{n^{3}}{6}(H \cdot H \cdot H)+\frac{n^{2}}{4}\left(H \cdot H \cdot K_{S}\right)+\frac{n}{12} \gamma+\chi\left(\mathcal{O}_{S}\right)
$$

for any $n \geqslant 1$. Put $h_{n}=h^{0}\left(\mathcal{O}_{S}(n H)\right)$. Then

$$
h_{4}-3 h_{3}+3 h_{2}-h_{1}=H \cdot H \cdot H
$$

and

$$
h_{3}-2 h_{2}+h_{1}=2(H \cdot H \cdot H)+\frac{1}{2}\left(H \cdot H \cdot K_{S}\right),
$$

which implies after applying (4.8)

$$
h_{4} \leqslant 2 h_{1}-5 h_{2}+4 h_{3}+1 .
$$

Since $H \cdot H \cdot H \geqslant 3$, the equality (4.9) also implies

$$
h_{4} \geqslant 3+h_{1}-3 h_{2}+3 h_{3} .
$$

Recall that $h_{1}=6, h_{2}=20, h_{3} \in\{20,36,56\}$.

If $h_{3}=20$, then (4.11) implies that $h_{4} \leqslant-12$, which is a contradiction.

If $h_{3}=36$, then (4.11) and (4.12) imply that $52 \geqslant h_{4} \geqslant 54$, which is a contradiction.

We see that $h_{3}=56$. Then (4.12) implies that $h_{4} \geqslant 114$, so that

$$
h_{4} \in\{120,126\}
$$

by (4.5). If $h_{4}=120$, then (4.9) implies that $H \cdot H \cdot H=9$. Note that

$$
H \cdot H \cdot H=h_{5}-3 h_{4}+3 h_{3}-h_{2},
$$

and hence $h_{5}=171$, which is impossible by (4.6). Thus, we see that $h_{4}=126$.

It follows from (4.9) and (4.13) that $H \cdot H \cdot H=15$ and $h_{5}=179$, which is impossible by (4.6). 


\section{Seven-dimensional singularities}

Let $G$ be a finite subgroup in $\mathrm{SL}_{7}(\mathbb{C})$, and let $\phi: \mathrm{SL}_{7}(\mathbb{C}) \rightarrow \operatorname{Aut}\left(\mathbb{P}^{6}\right)$ be the natural projection. Put $\bar{G}=\phi(G)$. We say that the subgroup $G$ is the lift of the subgroup $\bar{G}$ to $\mathrm{SL}_{7}(\mathbb{C})$.

Theorem 5.1 ([32, Theorem 4.1], [33, Theorem I]). Suppose that the subgroup $G$ is quasiprimitive. Then there is a lift of the subgroup $\bar{G}$ to $\mathrm{SL}_{7}(\mathbb{C})$ that is contained in the following list:

(I) a subgroup of the subgroup $G_{7} \subset \mathrm{SL}_{7}(\mathbb{C})$ such that

$$
G_{7}=\operatorname{Norm}_{\mathrm{SL}_{7}(\mathbb{C})}\left(\mathbb{H}_{7}\right) \cong \mathbb{H}_{7} \rtimes \mathrm{SL}_{2}\left(\mathbb{F}_{7}\right),
$$

where $\mathbb{H}_{7}$ is the Heisenberg group of order $7^{3}$, and the corresponding sevendimensional representation of the group $\mathbb{H}_{7}$ is any of its 7 -dimensional irreducible representations,

(II) $\mathrm{PSL}_{2}\left(\mathbb{F}_{13}\right)$,

(III) (i) $\mathrm{PSL}_{2}\left(\mathbb{F}_{8}\right)$,

(ii) an extension of the subgroup described in III(i) by an automorphism of order 3 ,

(IV) $\mathrm{A}_{8}$ or $\mathrm{S}_{8}$,

(V) (i) $\mathrm{PSL}_{2}\left(\mathbb{F}_{7}\right)$,

(ii) $\mathrm{PGL}_{2}\left(\mathbb{F}_{7}\right)$,

(VI) (i) $\operatorname{PSU}_{3}\left(\mathbb{F}_{3}\right)$,

(ii) an extension of the subgroup described in VI(i) by an automorphism of order 2 ,

(VII) $\operatorname{Sp}_{6}\left(\mathbb{F}_{2}\right)$.

Remark 5.1. Up to conjugation there are two primitive subgroups in $\mathrm{SL}_{7}(\mathbb{C})$ that are isomorphic to $\mathrm{PSL}_{2}\left(\mathbb{F}_{8}\right)$ : one is conjugate to a subgroup in $\mathrm{SL}_{7}(\mathbb{Q})$, and another is conjugate to a subgroup in $\mathrm{SL}_{7}\left(\mathbb{Q}\left(\xi_{9}\right)\right)$, where $\xi_{9}$ is a primitive root of unity of degree 9. Similarly, up to conjugation there are two primitive subgroups in $\mathrm{SL}_{7}(\mathbb{C})$ that are isomorphic to $\operatorname{PSU}_{3}\left(\mathbb{F}_{3}\right)$ : one is conjugate to a subgroup in $\mathrm{SL}_{7}(\mathbb{Q})$, another is conjugate to a subgroup in $\operatorname{SL}_{7}(\mathbb{Q}(\sqrt{-1}))$. The detailed information on the corresponding representations may be found in [10].

The main purpose of this section is to prove the following result.

Theorem 5.2. Suppose that $G$ is quasiprimitive. Then either $G$ has a semi-invariant of degree at most 7 , or $G$ is a subgroup of the subgroup $G_{7} \subset \mathrm{SL}_{7}(\mathbb{C})$ (see Theorem 5.1).

Proof. Recall that changing a lift of $\bar{G}$ to $\mathrm{SL}_{7}(\mathbb{C})$ does not change the degrees of semi-invariants, which implies that we may assume that $G$ is one of the groups listed in Theorem 5.1.

Note that the groups of type I have a unique lift to $\mathrm{SL}_{7}(\mathbb{C})$.

By Lemma 2.4, we may assume that $G$ is not conjugate to a subgroup in $\operatorname{SL}_{7}(\mathbb{Q})$, which implies that the subgroup $G$ is not of type IV, V(i) or VII (see [10]).

If the subgroup $G$ is of type II, III(i) or VI(i) (cf. Remark 5.1), then the minimal degree $d_{\min }$ of the invariants of the subgroup $G$ is given in the following table: 


\begin{tabular}{|c|c|c|c|}
\hline$G$ & $\mathrm{PSL}_{2}\left(\mathbb{F}_{13}\right)$ & $\mathrm{PSL}_{2}\left(\mathbb{F}_{8}\right)$ & $\mathrm{PSU}_{3}\left(\mathbb{F}_{3}\right)$ \\
\hline Type & $\mathrm{II}$ & $\mathrm{III}(\mathrm{i})$ & $\mathrm{VI}(\mathrm{i})$ \\
\hline$d_{\text {min }}$ & 2 & 2 & 3 \\
\hline
\end{tabular}

If the subgroup $G$ is a subgroup of type III(ii), then it has a normal subgroup of index 3 isomorphic to $\mathrm{PSL}_{2}\left(\mathbb{F}_{8}\right)$, which implies that $G$ has a semi-invariant of degree 2 by Lemma 2.5.

Arguing as in the case of a subgroup of type III(ii), we see that the subgroup $G$ has a semi-invariant of degree at most 3 if $G$ is a quasiprimitive subgroup of type $\mathrm{V}(\mathrm{ii})$ or VI(ii).

Proof of Theorem 1.5. By [22, Lemma 2.2.1(xi)] (see also [21, Section 1]), the group $G_{7} \subset \mathrm{SL}_{7}(\mathbb{C})$ has an invariant of degree 7 . Thus, Theorem 5.2 implies Theorem 1.5.

\section{Appendix A. Alpha-invariant}

Let $(V \ni O)$ be a germ of a Kawamata log terminal singularity (see [13, Definition 3.5]), and let $\pi: W \rightarrow V$ be a birational morphism such that

- the exceptional locus of $\pi$ consists of one irreducible divisor $E \subset W$ such that $O \in \pi(E)$,

- the log pair $(W, E)$ has purely log terminal singularities (see [13, Definition 3.5]),

- the divisor $-E$ is a $\pi$-ample $\mathbb{Q}$-Cartier divisor.

Theorem A.1. The birational morphism $\pi: W \rightarrow V$ does exist.

Proof. The required assertion follows from [23, Proposition 2.9], [14, Theorem 1.5] and [1].

The existence of $\pi$ is obvious if $(V \ni O)$ is a quotient singularity (see [7, Remark 3.15]) or an isolated quasihomogeneous hypersurface singularity.

Definition A.1 ([23, Definition 2.1]). We say that $\pi$ is a plt blow up of the germ of the singularity $(V \ni O)$.

Definition A.2 ([23, Definition 4.1]). We say that $(V \ni O)$ is weakly exceptional if $\pi$ is unique.

The goal of this appendix is to define an invariant $\alpha(V \ni O) \in \mathbb{R}$ of the singularity $(V \ni O)$, which is a local analog of the $\alpha$-invariant introduced in [30] and [31].

Lemma A.1 (see [23, Theorem 4.9]). If $(V \ni O)$ is exceptional, then one has $\pi(E)=O$.

Lemma A.2 ([14, Corollary 1.7],[1]). If $(V \ni O)$ is weakly exceptional, then one has $\pi(E)=O$. 
If $\pi(E) \neq O$, then we put $\alpha(V \ni O)=0$. Suppose, in addition, that $\pi(E)=O$.

Denote by $R_{1}, \ldots, R_{s}$ the irreducible components of the locus $\operatorname{Sing}(W)$ such that

$$
\operatorname{dim}\left(R_{i}\right)=\operatorname{dim}(V)-2
$$

and $R_{i} \subset E$ for every $i \in\{1, \ldots, s\}$. Put

$$
\Delta=\sum_{i=1}^{s} \frac{m_{i}-1}{m_{i}} R_{i}
$$

where $m_{i}$ is the smallest positive integer such that $m_{i} E$ is Cartier at a general point of the subvariety $R_{i} \subset E$. (One has $\Delta=\operatorname{Diff}_{E}(0)$ in the notation of the paper [23].)

Lemma A.3 ([13, Theorem 7.5]). The variety $E$ is normal. Moreover, the log pair $\left(E, \operatorname{Diff}_{E}(0)\right)$ is Kawamata log terminal.

The $\log$ pair $(E, \Delta)$ is a $\log$ Fano variety, i.e. the divisor $-\left(K_{E}+\Delta\right)$ is ample. Indeed, the divisor $-E$ is $\pi$-ample, and

$$
K_{E}+\left.\left.\Delta \sim_{\mathbb{Q}}\left(K_{W}+E\right)\right|_{E} \sim_{\mathbb{Q}}\left(\pi^{*}\left(K_{V}\right)+(1+a) E\right)\right|_{E} .
$$

Moreover, one has $a>-1$, because $V$ has Kawamata log terminal singularities. Put

$$
\operatorname{lct}(E, \Delta)=\sup \left\{\begin{array}{l|l}
\lambda \in \mathbb{Q} & \begin{array}{l}
\text { the log pair }(E, \Delta+\lambda D) \text { is log canonical } \\
\text { for any effective } \mathbb{Q} \text {-divisor } D \sim_{\mathbb{Q}}-\left(K_{E}+\Delta\right)
\end{array}
\end{array} .\right.
$$

Theorem A.2 ([14, Theorem 2.1]). The singularity $(V \ni O)$ is weakly exceptional if and only if the inequality $\operatorname{lct}(E, \Delta) \geqslant 1$ holds.

Note that the real number $\operatorname{lct}(E, \Delta)$ is an algebraic counter-part of the so-called $\alpha$-invariant introduced in [30] and [31] (cf. [6, Theorem A.3]). Put

$$
\alpha(V \ni O)=\left\{\begin{array}{l}
\operatorname{lct}(E, \Delta) \text { if } \operatorname{lct}(E, \Delta) \geqslant 1, \\
0 \text { if } \operatorname{lct}(E, \Delta)<1 .
\end{array}\right.
$$

Definition A.3. We say that $\alpha(V \ni O)$ is the alpha-invariant of the singularity $(V \ni O)$.

Note that $\alpha(V \ni O) \neq 0 \Longleftrightarrow \alpha(V \ni O) \geqslant 1 \Longleftrightarrow(V \ni O)$ is weakly exceptional.

Example A.1 ([5, Lemma 5.2]). Suppose that $(V \ni O)$ is an isolated quasihomogeneous hypersurface singularity

$$
z^{2} t+y t^{2}+x y^{4}+x^{8} z=0 \subset \mathbb{C}^{4} \cong \operatorname{Spec}(\mathbb{C}[x, y, z, t]),
$$

where $O \in V$ is given by $x=y=z=t=0$. Then $\alpha(V \ni O)=33 / 4$.

Theorem A.3 ([23, Theorem 4.9]). The the singularity $(V \ni O)$ is exceptional if and only if for every effective $\mathbb{Q}$-divisor $D$ on the variety $E$ such that $D \sim_{\mathbb{Q}}-\left(K_{E}+\Delta\right)$ the log pair $(E, \Delta+D)$ has Kawamata log terminal singularities.

Corollary A.1. The singularity $(V \ni O)$ is exceptional if $\alpha(V \ni O)>1$.

Corollary A.2. If $(V \ni O)$ is exceptional, then $(V \ni O)$ is weakly exceptional. 
Let $G$ be a finite subgroup in $\mathrm{GL}_{n+1}(\mathbb{C})$, where $n \geqslant 1$. Put

$$
\bar{G}=\phi(G) \subset \operatorname{Aut}\left(\mathbb{P}^{n}\right) \cong \operatorname{PGL}_{n+1}(\mathbb{C})
$$

where $\phi: \mathrm{GL}_{n+1}(\mathbb{C}) \rightarrow \operatorname{Aut}\left(\mathbb{P}^{n}\right)$ is the natural projection. Put

$$
\operatorname{lct}\left(\mathbb{P}^{n}, \bar{G}\right)=\sup \left\{\begin{array}{l|l}
\lambda \in \mathbb{Q} & \begin{array}{l}
\text { the log pair }\left(\mathbb{P}^{n}, \lambda D\right) \text { has log canonical } \\
\text { singularities for every } \bar{G} \text {-invariant effective } \\
\mathbb{Q} \text {-divisor } D \sim_{\mathbb{Q}}-K_{\mathbb{P} n}
\end{array}
\end{array}\right\} \in \mathbb{R} \text {. }
$$

Lemma A.4 ([7, Remark 3.2]). Suppose that $G$ does not contain reflections. Then

$$
\alpha(V \ni O)=\left\{\begin{array}{l}
\operatorname{lct}\left(\mathbb{P}^{n}, \bar{G}\right), \text { if } \operatorname{lct}\left(\mathbb{P}^{n}, \bar{G}\right) \geqslant 1, \\
0, \text { if } \operatorname{lct}\left(\mathbb{P}^{n}, \bar{G}\right)<1 .
\end{array}\right.
$$

We see that the number $\alpha(V \ni O)$ measures how exceptional $(V \ni O)$ is (cf. Remark 1.3).

Example A.2. Let $G \subset \mathrm{PSL}_{3}(\mathbb{C})$. Then

$$
\operatorname{lct}\left(\mathbb{P}^{n}, \bar{G}\right)=\left\{\begin{array}{l}
4 / 3, \text { if } \bar{G} \cong \mathrm{PSL}_{2}\left(\mathbb{F}_{7}\right), \\
2, \text { if } \bar{G} \cong \mathrm{A}_{6},
\end{array}\right.
$$

by [4, Examples 1.9 and 6.5$]$.

For a fixed $n \in \mathbb{Z}_{>0}$, the number $\operatorname{lct}\left(\mathbb{P}^{n}, \bar{G}\right)$ is bounded by Theorem 1.2 , because there exist only finitely many primitive finite subgroups in $\mathrm{SL}_{n+1}(\mathbb{C})$ up to conjugation (see [9]).

Theorem A.4 ([7, Theorem 1.24]). The inequality $\operatorname{lct}\left(\mathbb{P}^{n}, \bar{G}\right) \leqslant 4(n+1)$ holds for every $n \geqslant 1$.

In fact, we expect the following to be true (cf. [29]).

Conjecture A.1. There is a universal bound $\alpha \in \mathbb{R} \operatorname{such}$ that $\operatorname{lct}\left(\mathbb{P}^{n}, \bar{G}\right) \leqslant \alpha$ for any $\bar{G} \subset \operatorname{Aut}\left(\mathbb{P}^{n}\right)$ and $n \geqslant 1$.

One can try to tackle Conjecture A.1 using the classification of finite simple groups.

\section{Acknowledgments}

The authors would like to thank G. Robinson for numerous useful explanations and comments, and T. Dokchitser and A. Khoroshkin for computational support. The authors were partially supported by AG Laboratory GU-HSE, RF government grant no. 11 11.G34.31.0023. The first author was supported by the grant numbers NSF DMS-0701465 and EPSRC EP/E048412/1, the second author was supported by the grant numbers RFFI 08-01-00395-a, RFFI 11-01-00185-a, RFFI 11-01-00336a, N.Sh.-1987.2008.1 and N.Sh.-4713.2010.1. 


\section{References}

[1] C. Birkar, P. Cascini, C. Hacon and J. McKernan, Existence of minimal models for varieties of log general type, J. Amer. Math. Soc., 23(2) (2010), 405-468.

[2] W. Bosma, J. Cannon and C. Playoust, The Magma algebra system. I. The user language, J. Symbolic Comput. 24 (1997), 235-265.

[3] H. Blichfeldt, Finite collineation groups, University of Chicago Press, Chicago, 1917.

[4] I. Cheltsov, Log canonical thresholds of del Pezzo surfaces, Geom. Funct. Anal. 18 (2008), 1118-1144.

[5] I. Cheltsov, J. Park and C.Shramov, Exceptional del Pezzo hypersurfaces, J. Geom. Anal. 20 (2010), 787-816.

[6] I. Cheltsov and C.Shramov, Log canonical thresholds of smooth Fano threefolds, Russian Math. Surveys 63 (2008), 73-180.

[7] I. Cheltsov and C.Shramov, On exceptional quotient singularities, (2009), arXiv:math/0909.0918.

[8] C.-Y. Chi and S.-T. Yau, A new geometric approach to problems in birational geometry Proc. Natl. Acad. Sci. USA 105(48) (2008), 18696-18701.

[9] M. Collins, On Jordan's theorem for complex linear groups J. Group Theory, 10 (2007), 411-423.

[10] J. Conway, R. Curtis, S. Norton, R. Parker and R. Wilson, Atlas of finite groups, Clarendon, Oxford, 1985.

[11] Y. Kawamata, On Fujita's freeness conjecture for 3-folds and 4-folds, Math. Ann. 308 (1997), 491-505.

[12] Y. Kawamata, Subadjunction of log canonical divisors II, Amer. J. Math. 120 (1998), 893-899.

[13] J. Kollár, Singularities of pairs, Proc. Sympos. Pure Math. 62 (1997), 221-287.

[14] S. Kudryavtsev, On purely log terminal blow ups, Math. Notes 69 (2002), 814-819.

[15] R. Lazarsfeld, Positivity in algebraic geometry, II, Springer-Verlag, Berlin, 2004.

[16] J. Lindsey, On a projective representation of the Hall-Janko group, Bull. Amer. Math. Soc. 74 (1968), 1094

[17] J. Lindsey, On a six dimensional projective representation of the Hall-Janko group, Pac. J. Math. 35 (1970), 175-186.

[18] J. Lindsey, Finite linear groups of degree six, Canad. J. Math. 23 (1971), 771-790.

[19] O. Manz and Th. Wolf, Representations of solvable groups, London Math. Soc. Lecture Note Ser. 185, Cambridge University Press, 1993.

[20] D. Markushevich and Yu. Prokhorov, Exceptional quotient singularities, Amer. J. Math. 121 (1999), 1179-1189.

[21] F. Melliez and K. Ranestad, Degenerations of (1,7)-polarized abelian surfaces, Math. Scand. 97 (2005), 161-187.

[22] M. Miele, Klassifikation der Durchschnitte Heisenberg-Invariante Systeme von Quadriken in $\mathbb{P}^{6}$ PhD thesis, Erlangen, 1993.

[23] Yu. Prokhorov, Blow-ups of canonical singularities, Algebra (Moscow, 1998), de Gruyter, Berlin (2000), 301-317.

[24] Y.Prokhorov, Sparseness of exceptional quotient singularities, Math. Notes 68 (2000), 664-667.

[25] Y. Rubinstein, Some discretizations of geometric evolution equations and the Ricci iteration on the space of Kähler metrics, Adv. Math. 218 (2008), 1526-1565.

[26] G. Shephard, J. Todd, Finite unitary reflection groups, Canad. J. Math. 6 (1954), 274-304.

[27] V.Shokurov, Three-fold log flips, Russian Acad. Sci. Izv. Math. 40 (1993), 95-202.

[28] T.Springer, Invariant theory, Lecture Notes in Math. 585, Springer-Verlag, Berlin, New York, 1977.

[29] J. Thompson, Invariants of finite groups, J. Algebra 69 (1981), 143-145.

[30] G. Tian and On Kähler-Einstein metrics on certain Kähler manifolds with $\mathrm{c}_{1}(M)>0$, Invent. Math. 89 (1987), 225-246.

[31] G. Tian, S.-T. Yau, Kähler-Einstein metrics on complex surfaces with $\mathrm{C}_{1}>0$, Comm. Math. Phys. 112 (1987), 175-203.

[32] D. Wales, Finite linear groups of degree seven I, Canad. J. Math. 21 (1969), 1042-1056.

[33] D. Wales, Finite linear groups of degree seven II, Pac. J. Math. 34 (1970), 207-235. 
UNIVERSity OF EDINBURGH, EDINBURGH EH9 3JZ, UK

E-mail address: I.Cheltsov@ed.ac.uk

Steklov Institute of Mathematics, 8 Gubkina street, Moscow 119991, Russia

E-mail address: shramov@mccme.ru

Laboratory of Algebraic Geometry, GU-HSE, 7 Vavilova street, Moscow 117312, Russia 
